\title{
Editorials
}

\section{Artificial intelligence and diagnosis in general practice}

\section{INTRODUCTION}

Diagnosis in general practice is difficult. Within the populations that GPs care for, serious diseases and disorders are less prevalent and, generally, at an earlier stage in the development of symptoms than in other healthcare settings. ${ }^{1}$ However, the public, the media, and our specialist colleagues want primary care clinicians to become much better diagnosticians. GPs are now expected to spot individuals with cancer earlier while also avoiding excessive testing or inappropriate onward referrals.

Currently, there is growing interest in using artificial intelligence (Al) to supplement, enhance, or even replace GP diagnostic intelligence. Advocates of Al have suggested that such technologies could improve diagnostic accuracy (with fewer underdiagnoses and overdiagnoses) in addition to diagnostic efficiency. But others have argued that it will add yet another information burden during an already busy surgery. It might do very little to improve patient outcomes, GP stress levels, or healthcare finances. ${ }^{2}$

Al refers to computing techniques that resemble human cognitive functions, such as learning and problem solving. Rather than following pre-programmed instructions, Al approaches draw inferences from, or discover, patterns in data. Expert systems emulate human decision making by using an inference engine to apply rules to facts held in a knowledge base. Machine learning - including neural networks or deep learning - develops models to make decisions, or to classify examples. This is achieved by using a training algorithm - supplied with data of suitable quantity and quality - to adjust the weights within a model to optimise the goodness of fit between the input and the output.

All Al techniques depend on high-quality information on which to learn and classify clinical data in relation to outcomes. To date, most Al techniques have focused on radiology, as medical imaging data have been systematically stored and collected for some time and are now readily available

\section{there is growing interest in using Al to supplement, enhance, or even replace GP diagnostic intelligence.}

to train Al diagnostic systems. ${ }^{3}$ Based on an approach known as supervised learning, the programmer trains the system by defining a set of diagnoses for a range of images labelled by human experts.

Although clinical information from general practice is more ill defined, A natural language processing techniques now provide the tools to analyse more unstructured data from records or patients. The outputs are then in a form that is more amenable to further analysis by machine learning.

$\mathrm{Al}$ is a focused technology, and this is, on the face of it, at variance with the comprehensive and holistic nature of primary care medicine. Diagnostic intelligence in general practice is much more than generating a possible label or suggesting a course of action. ${ }^{1}$ The GP needs to understand the patient's context in addition to picking up social and psychological cues with empathy, care, and compassion. Explicit knowledge about the predictive value of symptoms can be taught to a machine but tacit knowledge such as how to gain an individual's confidence might not.

In this editorial we will consider some possible roles for $\mathrm{Al}$ to augment the current diagnostic approach within primary care settings.

\section{THE PATIENT'S PRESENTATION}

One of the challenges to improving health outcomes from conditions such as cancer depression, and rheumatoid arthritis is to get the right individuals to the right place at the right time. Before a person presents to their GP, they will often have sought advice from friends, their family, or the local pharmacist. Most will have also checked the internet. But the problem with all these interactions is that the advice received is of variable quality and consistency.

Several Al-based symptom checkers have been developed with the aim of improving the accuracy and the reliability of any advice, guidance, or signposting. In a vignette-based audit of 23 symptom checkers available for use by the public, the appropriate triage advice was considered to have been provided in $57 \%$ of evaluations, rising to $80 \%$ for emergency cases. ${ }^{4}$

Unfortunately, the major problem with most symptom checkers is that they are risk averse, encouraging users to seek help from their GP for conditions when self-care would be perfectly reasonable and safe. Striking a sensible balance between missing a serious condition and overwhelming GP surgeries is tricky. As many symptoms presenting to GPs are self-limiting, one option to consider is the development of Al tools that enable certain symptoms to be monitored over time. ${ }^{5}$ This symptom-based information might also be supplemented by clinical data provided directly by individuals using, for example, self-testing or wearable biosensors.

Images from an individual's mobile phone of a skin lesion can already be assessed by an Al system using deep learning to rule out skin cancers. This presents the opportunity to increase the numbers of individuals undergoing skin cancer screening and signpost the most appropriate ones to see their GP for further assessment. ${ }^{6}$

\section{THE GP CONSULTATION}

Clinical decision making is often partially dependent on a number of cognitive heuristics or 'rules of thumb' in order to aid recall or understand knowledge. Unfortunately, as a consequence, GPs may produce a distorted range of differential diagnoses through failing to consider the relative prevalences of conditions within a primary care setting. Data that contradict expectations also tend to be ignored or dismissed as unimportant, especially if obtained towards the end of the consultation.?

Within UK general practice the earlier 


\section{"Al could assist GPs in recognising and overcoming cognitive biases...}

\section{ADDRESS FOR CORRESPONDENCE}

Nick Summerton

Yorkshire Medical Chambers, 7 Hall Walk, Welton,

Brough, East Yorkshire HU15 1PN, UK.

Email: nsummertonঝdoctors.org.uk diagnosis of cancer continues to remain a challenge, and this might reflect the inappropriate use of certain heuristics. According to Sheringham et al, GPs are not more likely to initiate cancer investigations for individuals with higher-risk symptoms and, also, do not investigate everyone with the same symptoms equally. ${ }^{8}$

Al could assist GPs in recognising and overcoming cognitive biases, but only if the system has been trained on the correct data, and from the appropriate population. An Al diagnostic tool developed using clinical information from the UK might not provide the correct clinical guidance to a primary care clinician in India. Moreover, the Al outputs need to support GP decision making where, for example, precise diagnostic labels are less important than deciding on an appropriate course of action. ${ }^{1}$

In enhancing a GP's diagnostic abilities, it is also important to appreciate that Al systems do not get tired or irritable! Moreover, Al might have a key role in assessing and improving GP communication skills. ${ }^{9}$

More broadly, if we are really committed to improving the quality of primary care cancer diagnoses, then educating new GPs about cancer symptoms using retrospective studies of historical clinical data seems unlikely to have much impact on cancer prognoses. In order to significantly improve patient outcomes, we urgently need more and better information on the features that might indicate early-stage cancers within a primary care setting.

\section{CONCLUSION}

By its ability to analyse masses of data and to come up with new insights and associations, Al has the potential to furnish GPs with some fresh ideas and understandings. For example, it may highlight the potential importance of other non-traditional features in achieving an earlier cancer diagnosis, such as, measurable changes in a patient's lifestyle, or health-seeking behaviour when they consult with a non-specific symptom such as fatigue. ${ }^{10}$
In addition, Al might also point GPs towards new diagnostic approaches, for example, the analysis of an individual's social media data history to assist in the earlier recognition of depression. ${ }^{11}$

The clinical information required to distinguish between, for example, two courses of action in general practice is distinct from that required to make a precise clinical diagnosis in a specialist setting. In keeping with this, it is of some concern that the number of tests being ordered by GPs has risen over three-fold between 2000 and 2015. ${ }^{12}$ Such an increase will have adverse effects on patients in addition to healthcare finances. Therefore, when faced with some common symptoms - such as tiredness or fatigue - it might be that Al can be harnessed in order to support more rational approaches to testing within general practice. ${ }^{13}$

To conclude, Al cannot replace GP diagnostic intelligence, but could augment it. However, before any large-scale rollout is considered, there is a requirement for Al systems to be subjected to careful evaluation. ${ }^{14}$ We need to ensure that the Al-GP combination enhances diagnostic decision making and health outcomes without simply producing anxious patients or inflicting a further burden on healthcare budgets.

\section{Nick Summerton,}

GP, Yorkshire Medical Chambers, Welton, Brough, East Yorkshire.

\section{Martin Cansdale,}

Principal Data Scientist, Your.MD Limited, Bloomsbury, London.

\section{Provenance}

Freely submitted; externally peer reviewed.

\section{Competing interests}

Nick Summerton sits on the Clinical Advisory Group for Your.MD Ltd. Martin Cansdale is employed by Your.MD Ltd. Your.MD Ltd has developed an Al-based symptom checker.

DOI: https://doi.org/10.3399/bjgp19X704165

\section{REFERENCES}

1. Summerton N. Primary care diagnostics. Abingdon: Radcliffe Medical Press, 2011.

2. Academy of Medical Royal Colleges. Artificial intelligence in healthcare. London: AoMRC, 2019.

3. Tang A, Tam R, Cadrin-Chênevert A, et al. Canadian Association of Radiologists White Paper on artificial intelligence in radiology. Can Assoc Radiol J 2018; 69(2): 120-135.

4. Semigran HL, Linder JA, Gidengil C, Mehrotra A. Evaluation of symptom checkers for self diagnosis and triage: audit study. BMJ 2015; 351: h3480.

5. Kroenke K, Jackson JL. Outcome in general medical patients presenting with common symptoms: a prospective study with a 2-week and a 3-month follow-up. Fam Pract 1998; 15(5): 398-403

6. Zakhem GA, Motosko CC, Ho RS. How should artificial intelligence screen for skin cancer and deliver diagnostic predictions to patients? JAMA Dermatol 2018; 154(12): 1383-1384.

7. Tversky A, Kahneman D. Judgement under uncertainty: heuristics and biases. Science 1974; 185(4157): 1124-1131.

8. Sheringham J, Sequeira R, Myles J, et al. Variations in GPs decisions to investigate suspected lung cancer: a factorial experiment using multimedia vignettes. BMJ Qual Saf 2017; 26(6): 449-459.

9. Ryan P, Luz S, Albert P, et al. Using artificial intelligence to assess clinicians' communication skills. BMJ 2019; 364: 1161.

10. Gray DJP. The role of the general practitioner in the early detection of malignant disease. Trans Hunterian Soc 1966; 25: 135-179.

11. Eichstaedt JC, Smith RJ, Merchant RM, et al. Facebook language predicts depression in medical records. Proc Natl Acad Sci U S A 2018; 115(44): 11203-11208.

12. [No authors listed]. Temporal trends in use of tests in UK primary care, 2000-15: retrospective analysis of 250 million tests. BMJ 2019; 364: 1444 .

13. Koch $H$, van Bokhoven MA, ter Riet $G$, et al. Ordering blood tests for patients with unexplained fatigue in general practice: what does it yield? Results of the VAMPIRE trial. Br J Gen Pract 2009; DOI: https://doi. org/10.3399/bjgp09X420310.

14. Fraser H, Coiera E, Wong D. Safety of patientfacing digital symptom checkers. Lancet 2018; 392(10161): 2263-2264. 